Gynäkologe 2021 · 54:307-308

https://doi.org/10.1007/s00129-021-04797-2

Angenommen: 30. März 2021

(c) Springer Medizin Verlag GmbH, ein Teil von Springer Nature 2021

\author{
Nicolai Maass ${ }^{1}$. Wolfgang Janni ${ }^{2}$ \\ 'Campus Kiel, Klinik für Gynäkologie und Geburtshilfe, Universitätsklinikum Schleswig Holstein, Kiel, \\ Deutschland \\ ${ }^{2}$ Frauenheilkunde und Geburtshilfe, Universitätsklinikum Ulm, Ulm, Deutschland
}

\title{
Das Mammakarzinom im Fokus
}

\section{Evolution von einer Therapie für alle zur hochspezifischen Individualtherapie}

Auch wenn sich die Welt in der COVID19-Pandemie befindet, stellt das Mammakarzinom weiterhin die häufigste Krebserkrankung der Frau dar. Befürchtungen, dass es zu Verzögerungen im Screening und in der Diagnostik des Mammakarzinoms während der Pandemie kommen könnte, mit einer daraus resultierenden Verschiebung der Tumorstadien bei Erstdiagnose, scheinen sich im angenommenen Ausmaß nicht zu bestätigen. Toral Gathani et al. berichten eine lediglich um $16 \%$ gegenüber dem Vorjahr reduzierte Rate an Erstbehandlungen im ersten Halbjahr 2020 im Rahmen einer Auswertung der Daten des National Health Service in England. Welche langfristigen Folgen jedoch durch die Pandemie auf die Diagnostik und Therapie des Mammakarzinoms und die daraus resultierende Prognose unserer Patientinnen entstehen werden, gilt es abzuwarten.

Durch die Aufteilung des Mammakarzinoms in die entsprechenden Subtypen, klinisch definiert anhand des Östrogen-, Progesteron- und HER2-Rezeptorstatus in Kombination mit dem Proliferationsmarker Ki67 ist schon in der adjuvanten Situation eine enorme Therapieindividualisierung entstanden. Während noch vor einigen Jahren Patientinnen mit einem klinisch hohen Rezidivrisiko in groß angelegte adjuvante und neoadjuvante Chemotherapiestudien relativ unabhängig von ihrem Rezeptorstatus eingeschlossen wurden, so werden inzwischen immer spezifischere Studien für die einzelnen Subtypen des Mammakarzinoms designt.
Die Frage der Definition des Rezidivrisikos anhand von klinischen Parametern oder mittels Zuhilfenahme von genomischen Tests steht insbesondere in Bezug auf das hormonrezeptorpositive Mammakarzinom bei der Entscheidung zwischen alleiniger endokriner Therapie oder chemoendokriner Therapie derzeit klar im Fokus der Diskussion. In der metastasierten Situation dieser Erkrankung haben die endokrinen Kombinationstherapien mit den potenten CDK4/6-Inhibitoren längst die Chemotherapien als First-line-Therapie ersetzt.

Dennoch bleiben weiterhin die Fragen der endokrinen Resistenz ein Thema der Forschung insbesondere in Bezug auf die assoziierten Mutationen z.B. im ESR1- und PIK3CA-Gen. Mit Alpelisib als PI3-Kinaseinhibitor wurde die erste zielgerichtete Substanz zugelassen, der eine somatische Mutation des Mammakarzinoms als Therapievoraussetzung zugrunde liegt.

Beim triple-negativen Mammakarzinom ist es insbesondere die Immunonkologie, die einen entscheidenden Beitrag zur Erweiterung der Therapievielfalt dieser prinzipiell mit einem schlechten Outcome in der metastasierten Situation behafteten Erkrankung geleistet hat. Dennoch gilt es, die Chemotherapiekombinationspartner und geeignete Subkohorten von Patientinnen mit therapeutischem Benefit noch besser zu evaluieren. Ob die Immunonkologie auch in der neoadjuvanten Behandlung des triple-negativen Mammakarzinoms zum Standard werden wird, ist in den kommenden Jahren abzuwarten. 
Insbesondere die HER2-gerichtete Therapievielfalt konnte in den letzten Jahren enorm erweitert werden. Viele vielversprechende Substanzen, Antikörper-Drug-Konjugate und Tyrosinkinasehemmer, haben ihre Wirksamkeit bereits in der metastasierten Situation bewiesen und erweitern die zur Verfügung stehende Therapieauswahl in den entsprechenden Therapielinien. Extensive Studienprogramme rekrutieren bereits oder befinden sich in Planung, um den Stellenwert diese Substanzen auch in der Primärtherapie zu etablieren.

Die Verfügbarkeit der Techniken zur molekularbiologischen Charakterisierung der Karzinome eröffnet eine neue Ebene der Tumortherapie, weg von der Entität zur mutationsspezifischen Therapie. Dies verlangt von uns Therapeuten die Etablierung von molekularen Tumorboards, die vielerorts schon entstanden sind oder sich im Aufbau befinden. In den sog. Basket-Trials, z. B. dem I-SPY2Programm, werden bereits einzelne Substanzen mutationsspezifisch untersucht, mit vielversprechenden Resultaten. Die Umsetzung in die Routine gilt es in Zukunft aktiv zu gestalten.

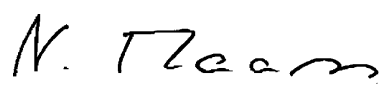

Prof. Dr. Nicolai Maass

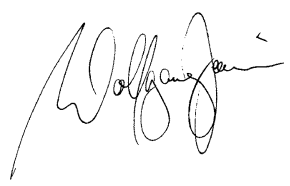

Prof. Dr. Wolfgang Janni

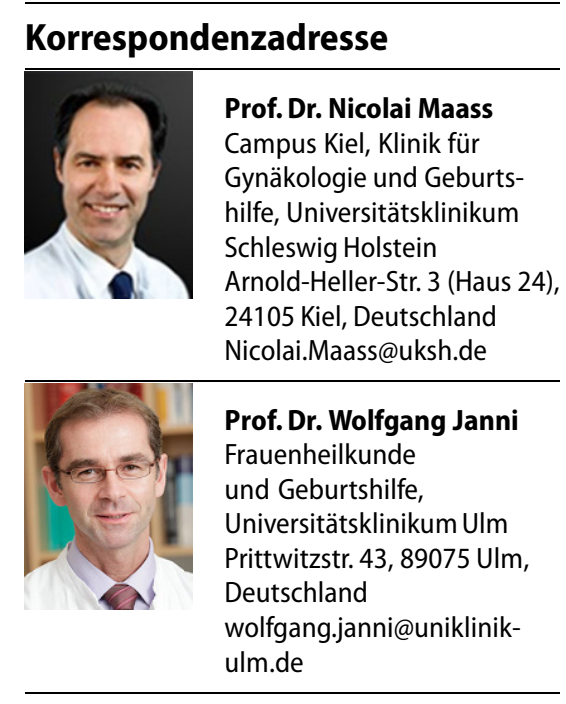

Hier steht eine Anzeige. Springer

Interessenkonflikt. N. Maass und W. Janni geben an, dass kein Interessenkonflikt besteht. 